Archives de sciences sociales des religions

$168 \mid 2014$

Bulletin Bibliographique

$\mathrm{Du}$ « chamanisme primitif »

Réponse à Françoise Aubin

\title{
Charles Stépanoff
}

\section{CpenEdition}

Journals

Édition électronique

URL : http://journals.openedition.org/assr/25858

DOI : $10.4000 /$ assr.25858

ISSN : $1777-5825$

Éditeur

Éditions de l'EHESS

Édition imprimée

Date de publication : 31 décembre 2014

Pagination : 93-102

ISBN : 978-2-7132-2467-6

ISSN : 0335-5985

Référence électronique

Charles Stépanoff, «Du « chamanisme primitif » », Archives de sciences sociales des religions [En ligne], 168 | 2014, mis en ligne le 11 mai 2015, consulté le 21 avril 2019. URL : http://

journals.openedition.org/assr/25858

Ce document a été généré automatiquement le 21 avril 2019.

(c) Archives de sciences sociales des religions 


\title{
Du « chamanisme primitif»
}

\author{
Réponse à Françoise Aubin
}

\section{Charles Stépanoff}

1 Françoise Aubin a consacré à notre ouvrage, Le chamanisme de Sibérie et d'Asie centrale, coécrit avec Thierry Zarcone, un compte rendu qui, quoique dans l'ensemble élogieux, formule une critique sévère à propos du traitement du chamanisme sibérien (ASSR 2013, $\left.164^{1}\right)$. Les reproches qui me sont adressés, puisque je suis l'auteur de ces passages, sont émaillés d'inexactitudes, d'imprécisions et de stéréotypes. On pourrait mettre ces erreurs sur le compte d'une lecture trop rapide, cependant les positions sur le chamanisme qui y sont exprimées sont révélatrices de partis pris évolutionnistes qui appellent une réponse ${ }^{2}$

2 Après avoir qualifié notre ouvrage de "bijou de pédagogie ", résumé ses différentes parties et salué ses points forts, l'auteur du compte rendu relève un "point faible", également qualifié d'« oubli », dont nous citerons in extenso l'exposé : «Le chamanisme primitif des chasseurs est bien négligé (le mot "chasse" n'apparait d'ailleurs pas dans l'index). Hors un bon exposé sur la cure assumé [sic] par le chamane musulman (p. 77-81), seul le mort est cité comme interlocuteur du chamane dans le monde invisible (p. 76). Or l'on sait bien, depuis le livre bouleversant de Roberte Hamayon, La chasse à l'âme de 1990 ( cf. ASSR, 74-256), que le chamanisme sibérien le plus anciennement attesté repose sur des rapports réciproques, égalitaires et horizontaux entre le chamane représentant le chasseur et son interlocuteur dans l'au-delà figurant les âmes du gibier, des rapports scellés par une alliance matrimoniale sur le modèle épique et qui donnent accès au gibier. L'introduction de l'élevage et la stratégie d'investissement qui en a découlé ont fait, seulement alors, basculer à la verticale la conception des rapports avec l'au-delà: l'interlocuteur invisible est désormais l'ancêtre.»

3 Commençons par les critiques de détail qui nous sont adressées. Il est difficile de comprendre pourquoi Aubin affirme que «seul le mort est cité comme interlocuteur du chamane dans le monde invisible ». En réalité, outre le dialogue avec l'âme du défunt, nous consacrons plusieurs pages (p. 58-61) dans le chapitre « Le monde du chamane » à la description de «l'armée des esprits en Sibérie », parmi lesquels nous décrivons le cervidé, 
l'ours, le plongeon, le serpent, la grenouille, etc. Nous citons à titre d'exemple les esprits d'un chamane chor, parmi lesquels la fille-esprit maitresse du tambour: "Cette filleesprit était l'épouse céleste du chamane, une idée typique des sociétés de chasseurs qui valorisent l'union matrimoniale entre les hommes et les esprits.» (p.59) Le thème de l'alliance matrimoniale et son lien avec la chasse n'est donc nullement «oublié » et le choix de ce chamane chor fait évidemment écho aux pages de La chasse à l'âme sur la tradition chore du mariage du chamane avec son tambour représentant la fille du dieu Ülgen (Hamayon 1990, 459-461).

4 Le résumé de La chasse à l'âme de Roberte Hamayon par Françoise Aubin suscite une série de questions tant sur le plan terminologique que théorique. Le chamane aurait, selon Aubin, un «interlocuteur dans l'au-delà ». Or la notion d'" au-delà » désigne dans $L a$ chasse à l'âme le monde des morts (p.276, p.685), et Hamayon critique précisément la position de W. Heissig (Heissig 1987) selon laquelle la quête du héros épique représenterait le voyage du chamane dans l'au-delà en quête d'âmes de morts : en effet, explique Hamayon, « la notion de ramener des âmes du monde des morts ne paraît pas fondamentale dans le chamanisme ", elle est même « exclue du discours lié à la pratique chamanique » (p. 276).

Dans son exposé, Aubin présente La chasse à l'âme comme un essai historique sans nuance affirmant sur un mode évolutionniste le passage d'un "chamanisme primitif » à une forme dérivée. Signalons tout d'abord qu'on ne trouve évidemment dans l'ouvrage de Hamayon aucune occurrence de cette notion surannée de « chamanisme primitif ». Et en effet, c'est un contraste entre des idéologies et non une succession de stades historiques que Hamayon décrit, se situant dans une perspective anthropologique. Hamayon le souligne avec clarté: "La Sibérie offre l'image d'un continuum dans lequel toute classification a quelque chose d'arbitraire. Aussi y a-t-il quelque artifice à isoler un point de vue de chasseur. Néanmoins, ce n'est pas à cerner des profils de sociétés historiques que l'on s'attache ici, mais à saisir des liens entre mode de vie et mode de pensée. » (ibid. p. 326-327)

6 Laissant à Françoise Aubin la responsabilité de son interprétation de La chasse à l'âme, nous nous bornerons ici à justifier les positions soutenues dans notre petit ouvrage $L e$ chamanisme de Sibérie et d'Asie centrale. Aubin affirme et nous reproche d'ignorer que le " chamanisme sibérien le plus anciennement attesté » a deux caractères notables : $1 /$ il est antérieur à "l'introduction de l'élevage ", 2/il se caractérise par des rapports « réciproques, égalitaires et horizontaux » entre le chamane et les esprits.

7 À quelle époque devraient remonter les attestations disponibles de pratiques chamaniques pour satisfaire au premier critère? Autrement dit, à quelle époque remonte la présence de l'élevage en Sibérie?

8 En Asie septentrionale, les premières traces d'élevage appartiennent à la culture d'Afanasiev, qui, entre 3700 et 2700 av. J.-C., s'étendait sur les hauteurs de l'Altaï et dans les vallées du Ienisseï et de l'Irtich. L'élevage y était déjà la ressource principale : sur les sites de l'Altaï, les restes fauniques mis au jour sont à $70 \%$ d'origine domestique contre $30 \%$ d'origine sauvage (Frachetti 2012 ; Luneau 2013). Plus au nord, dans la vallée de Minoussinsk (moyen Ienisseï), on trouve également des ossements de moutons et de chevaux datant du quatrième millénaire (Svitkova 1993). Des pétroglyphes de cette même région, datant des $\mathrm{III}^{\mathrm{e}}-\mathrm{II} \mathrm{e}^{\mathrm{e}}$ millénaires av. J.-C., représentent des charriots tirés par des bovins (Esin 2012). Il y a environ 3000 ans, des pasteurs nomades sont établis en 
Cisbaïkalie (Sagan-Zaba) (Nomokonova et al. 2010). En Transbaïkalie, le fort d'Ivolga, près d'Oulan-Oude, datant des $\mathrm{III}^{\mathrm{e}}-\mathrm{II} \mathrm{e}^{\mathrm{e}}$ siècles av. J.-C. était occupé par une population xiong-nu pratiquant l'élevage et l'agriculture : on y a retrouvé des ossements d'ovins et de caprins ainsi que des réserves de céréales (M. L. Machicek and A. V. Zubova 2012). Si le bétail a eu originellement au néolithique pour seule fonction la production de viande, l'utilisation des produits secondaires est manifestée à l'âge du bronze par les fromages découverts dans le kourgane de Pazyryk II, dans l'Altaï (Potapov 1953).

Cet élevage ancien concerne les zones méridionales steppiques de la Sibérie. Dans les régions septentrionales de taïga et de toundra, l'animal dont l'élevage est le plus ancien et le plus étendu est le renne (rangifer tarandus). Classiquement, la domestication du renne a été datée par les spécialistes soviétiques de la fin du $\mathrm{I}^{\mathrm{er}}$ millénaire av. J.-C. (Vasilevič and Levin 1951). Dans le bassin du moyen Ienisseï, le grand pétroglyphe de Boyar (III ${ }^{\mathrm{e}} \mathrm{II}{ }^{\mathrm{e}}$ siècles av. J.-C.) représente un village environné de troupeaux de bovins et de rennes, ainsi que de cavaliers montant chevaux et rennes (Kyzlasov 1952). Dans le nord, un élément d'attelage de renne trouvé à Ust' Poluj et daté du tournant de notre ère serait la trace la plus ancienne d'élevage samoyède (Golovnev 2004, 31, 72). Sans donner de précisions nouvelles en matière de chronologie, des études génétiques récentes confirment l'existence d'un foyer de domestication du renne en Sibérie distinct du foyer scandinave (Røed et al. 2008).

10 Qu'en est-il des premières sources sur le chamanisme sibérien actuellement disponibles pour le chercheur? Chronologiquement très postérieures aux premiers foyers d'élevage connus en Sibérie, elles se situent dans des sociétés où l'élevage est clairement établi comme une activité majeure de la vie quotidienne. Malgré son format succinct, nous avons tâché dans notre ouvrage d'offrir au lecteur un aperçu des sources historiques, qui a probablement échappé à l'attention de Françoise Aubin. Dans la section « Relations de voyage », nous citons sous l'intitulé « La plus ancienne description de chamane sibérien » un récit datant de 1556 par Richard Johnson. Dans cette description, un chamane des Samoyèdes de la Petchora ordonne au cours d'un rituel la mise à mort de cinq rennes : le lecteur informé peut en déduire que cette population dispose de troupeaux de rennes domestiques, immédiatement disponibles pour un sacrifice. C'est ce que confirme le reste du texte non cité dans notre ouvrage: les Samoyèdes de la Petchora rencontrés par Johnson se déplacent en traîneaux tirés par des rennes et le rituel qu'il décrit est un sacrifice accompli dans chaque famille à l'occasion des nomadisations (Johnson 1886). La mobilité des éleveurs de rennes dans l'Arctique étant particulièrement forte, de tels sacrifices réguliers ne devaient être possibles que grâce à la possession d'importants troupeaux destinés à la production de viande et non seulement au trait. En effet, si les Samoyèdes avaient dû abattre leurs animaux de trait, ils auraient perdu leurs moyens de transport. Ainsi, la description la plus ancienne de chamanisme sibérien se situe très clairement dans une société où, si la chasse est probablement pratiquée, l'élevage occupe une place centrale, sous une forme qui n'est guère éloignée du pastoralisme.

11 Voyons maintenant la célèbre description d'un chamane toungouse rencontré par l'archiprêtre Avvakum en 1661 : «ce manant de magicien, près de ma cabane, amena sur le soir un bélier vivant et se mit à pratiquer sa magie : après l'avoir tourné et retourné, il lui tordit le cou et rejeta sa tête au loin.» (Avvakum 1938, 128-129). Dans ce témoignage d'Avvakum, comme dans celui de Johnson, le rituel observé est un sacrifice d'animal, une action qui témoigne de la domination et du contrôle de l'humain sur le bétail, et sans doute pas d'un modèle de réciprocité horizontale entre humains et non-humains. La 
possibilité d'accomplir communément des sacrifices indique que les animaux ne sont pas utilisés seulement comme des partenaires de travail, comme c'est le cas dans les petits élevages de rennes forestiers (Stépanoff 2012), mais que le bétail est utilisé comme une réserve vivante de viande (Ingold 1974).

Citons une dernière description ancienne : à la fin du XVII ${ }^{e}$ siècle, Isbrand Ides rencontra un chamane toungouse et fit cette observation: "Le magicien avait beaucoup de troupeaux qui venaient de ce qu'ils lui donnent toujours tout ce qu'il demande. » (Ides 1704, 30) À nouveau, le contexte économique est clairement marqué par un élevage qui n'a rien de marginal.

Pour trouver des sources écrites plus anciennes concernant le chamanisme, il faut se diriger vers l'Asie centrale. L'historien byzantin Ménandre décrit chez les Turcs occidentaux en 568 un rituel de purification accompli par des spécialistes rituels utilisant des tambours et «tombant en frénésie » pour chasser les mauvais esprits (Potapov 1991, 121). Or avec les Turcs anciens, on se situe dans un monde de pastoralisme nomade, plus dominé encore que les sociétés citées précédemment par les impératifs de l'élevage de bétail.

14 Les vestiges archéologiques sont-ils plus loquaces que les sources écrites et nous livreront-ils enfin des indices d'un chamanisme "primitif " antérieur à l'élevage? Roberte Hamayon a critiqué les théories récentes qui, dans un contexte de fascination occidentale croissante pour les pratiques chamaniques, attribuent aux chasseurs préhistoriques un chamanisme universel et primordial (Hamayon 2001). Les traditions chamaniques, fondées sur la mémorisation de chants et d'images, laissent évidemment peu de traces et offrent matière à l'imagination. En Sibérie, de nombreux pétroglyphes zoomorphes préhistoriques ornant les parois rocheuses des bassins du Ienisseï, du Baïkal et de l'Amour ont été interprétés par certains chercheurs comme des signes magiques attestant un chamanisme ancien. Cependant Henri-Paul Francfort a montré la fragilité de ces interprétations fondées sur une absence de définition rigoureuse de la notion de chamanisme : «It is (...) not before the second half of the first millenium A. D. that we can recognize shamanism and shamans in Turkic petroglyphs, as we know them from current ethnographic data: dancing characters holding tambourines at Tamgaly (Kazakhstan) and Sukhanikha (Khakassia), and more in later petroglyphs in Altaï and Tuva. Literary sources also now become available. For earlier times, one may not expect to determine more than some prehistoric societies of shamanic type. Otherwise we may well find that "shamanism" is the universal default religion, in a vague sense, in which we may also lose the consistency of the discourse. If that is so, then "shamanism" will acquire in our discipline the same status that "hunting magic", "animism" and "totemism" had at the beginning of the last century. » (Francfort 2001, 260-261)

15 Les pétroglyphes de Tamgaly et de Sukhanikha sont l'œuvre de populations turcophones pastorales, ce qui nous situe encore une fois dans le monde de l'élevage. L'opinion de Francfort concorde avec celle de l'archéologue Esther Jacobson qui, en outre, situe dans l'Altaï turcophone l'origine du chamanisme (Jacobson 2001, 291 ; cf. Jacobson 2004). Ce sont les conclusions de ces auteurs qui nous ont menés à indiquer dans notre ouvrage : "Selon les études archéologiques les plus rigoureuses, le chamanisme Nord asiatique, défini comme une institution centrée sur des spécialistes rituels, a probablement émergé dans la région de l'Altaï turc au cours du premier millénaire de notre ère. » (p. 15) Évitant en effet de confondre animisme et chamanisme, nous définissons ce dernier de façon empirique et sociologique comme une institution accordant à des spécialistes sur 
d'importantes actions rituelles un monopole que garantit d'une part par l'attribution de particularités corporelles héréditaires et d'autre part la possession d'un équipement distinctif complexe (tambour, costume...).

17 L'hypothèse d'une origine méridionale de l'institution chamanique entendue en ce sens strict, parmi les populations altaïques aux confins de la forêt sibérienne et des steppes de haute Asie, trouve dans les régions septentrionales d'intéressants indices de confirmation. Chez un peuple chasseur de l'Arctique comme les Ioukaghirs, qui n'ont jamais connu qu'un élevage de rennes très marginal, W. Jochelson nota à la fin du XIX siècle que le costume chamanique y était d'apparition récente. Les costumes qu'il a pu observer étaient empruntés aux Iakoutes et aux Toungouses. Avec ces costumes sont apparus des chamanes professionnels qui devinrent les seuls détenteurs légitimes des tambours rituels, alors que par le passé chaque famille ioukaghire disposait d'un tambour, comme chez les Tchouktches et les Koriaks (Jochelson 1926, 168-169). Ce monopole gagné par les chamanes est clairement le résultat d'une influence des peuples altaïques. De même, E. Alekseenko, spécialiste des Ket, peuple chasseur de Sibérie centrale, considère que le tambour chamanique de cette ethnie doit son origine aux contacts entre Ket et peuples turco-mongols au début du XVII ${ }^{\mathrm{e}}$ siècle (Alekseenko 1984, 82).

18 Quoi qu'il en soit de cette hypothèse, que nous ne pouvons développer pleinement ici, nous avons vu que, contrairement à ce que suppose Aubin, les premières attestations du chamanisme sibérien se situent dans des sociétés qui connaissent l'élevage depuis de nombreux siècles. Certes l'ethnographie plus tardive du chamanisme des peuples chasseurs est d'une grande richesse, mais il convient à ce sujet de prendre garde aux faux archaïsmes et de noter que l'insertion d'un fait ethnographique dans un contexte cynégétique n'est pas une preuve d'ancienneté. Ainsi l'identification d'un peuple comme " chasseur " n'implique pas qu'il n'ait pas connu l'élevage antérieurement. On est enclin à n'imaginer de transition possible que de la chasse vers l'élevage, or en réalité, les cas n'ont pas été exceptionnels en Sibérie, à la suite de bouleversements écologiques, d'épizooties ou de changements politiques, de passage d'une économie d'élevage à une économie de chasse (Takakura 2012 ; Stépanoff et al. 2013).

L'absence d'attestation ancienne est bien entendu indépendante de la question des liens fondamentaux qui peuvent unir le chamanisme et la chasse sur le plan théorique. Mais il n'était pas dans l'intention de cet ouvrage de vulgarisation de proposer une interprétation théorique des fondements du chamanisme et moins encore d'en désigner des traits purs et primordiaux aux dépens de traits superficiels. Il s'agissait plutôt de sélectionner pour leur représentativité des traits récurrents manifestant une forte stabilité malgré la diversité des contextes sociaux et historiques où ils ont été relevés. Nous nous sommes ainsi appuyés sur des traditions chamaniques insérées dans les pratiques d'élevage et de chasse, mais aussi sur les formes contemporaines qui se sont épanouies en contexte post-communiste sédentaire et urbain, largement documentées par la recherche récente (Merli 2010 ; Humphrey 1999 ; Pedersen 2011 ; Hamayon 2007 ; Stépanoff 2014).

20 Ainsi, Françoise Aubin regrette que, parmi les exemples de rituels sibériens que nous décrivons, figure la communication du chamane avec un défunt, pratique qui ne lui semble probablement pas représentative du chamanisme " primitif » : or ce choix ne doit rien au hasard. La fonction de psychopompe est, avec celle de thérapeute, une des plus communes des chamanes d'Asie septentrionale. Le chamane conduit l'âme du défunt vers 
le monde des morts chez les Touvas, les Tofalars, les Téléoutes, les Altaïens, les Chors, les Khakasses (Stépanoff 2014); dans le monde toungouso-mandchou, ce rituel, dont le rôle central dans la société nanaï est bien connu (Lopatin 1960 ; Delaby 1998), était aussi une attribution des chamanes chez les Evenks (Vasilevič 1969; Shirokogoroff 1935) et il est également décrit chez un peuple samoyède comme les Enets (Prokof'eva 1953, 210).

Les expressions de Françoise Aubin "son interlocuteur", "l'interlocuteur", sousentendent que le chamane ne s'adresse qu'à un seul esprit. Toutes les ethnographies montrent au contraire que les chamanes nomment dans leurs chants des esprits divers, nombreux comme des «nuées de moustiques » ou comme " une armée ». Ainsi il est peu probable que l'on puisse découvrir dans l'ethnographie une seule occurrence à l'état pur de l'une ou de l'autre des deux formes de chamanisme que F. Aubin fait se succéder. Je n'ai pas connaissance d'une tradition chamanique en Sibérie dans laquelle les chamanes n'auraient de rapport qu'avec des animaux et aucun avec des ancêtres ou au contraire dans laquelle seuls les ancêtres seraient invoqués sans aucun emprunt à la zoosémiotique. De même, la complexité des ethnographies offre peu d'appui empirique à l'affirmation par Aubin d'un passage mécanique de relations "horizontales" chez les chasseurscollecteurs à des relations "verticales" chez les pasteurs. La simple lecture des informations apportées dans notre ouvrage, si modeste soit-il, aurait pu inciter à plus de prudence. Nous montrons p. 55-56 que l'ascension du chamane dans le ciel était figurée chez les Nganassanes, les Evenks, les Téléoutes, les Selkoupes par un arbre ou un poteau. Notre iconographie en fournit même des exemples issus des Evenks et des Selkoupes. Or Evenks, Selkoupes, Nganassanes figurent incontestablement parmi les peuples dont les économies sont, à l'époque où ces rituels ont été relevés, dominées par la chasse.

À vrai dire, il est bien difficile, sur le plan historique, d'attribuer une origine récente à la représentation verticale d'un univers disposé en étages tant ses attestations sont anciennes et étendues. Dans toutes les traditions chamaniques turcophones, c'est le même terme kat/hat (en iakoute xattygas) qui désigne les étagesdu ciel que parcourt le chamane. Or un texte de Tourfan en langue turque ancienne ( $\mathrm{VIII}^{\mathrm{e}}-\mathrm{IX}^{\mathrm{e}} \mathrm{s}$. ap. J.-C.) le mentionne déjà dans ce sens : on qat kök tegri, « le ciel bleu à dix étages » (Borovkova 1969, 432).

En réalité, dans la pragmatique des rituels chamaniques, l'horizontalité et la verticalité ne sont pas deux étapes successives d'une évolution, mais deux dimensions d'un même système topologique. Le cadre spatial de l'action rituelle s'appuie sur une coordination précise entre ces plans qui permet de penser la verticalité du voyage chamanique à travers l'espace virtuel au sein de l'horizontalité de l'espace réel (Stépanoff 2013).

Trop souvent, on associe comme une évidence le chamanisme à une supposée " primitivité » intemporelle sans qu'il soit nécessaire d'appuyer ces intuitions sur des témoignages historiques ou archéologiques. L'un des buts de notre petit ouvrage était de montrer qu'une approche scientifique se doit d'être plus exigeante. 


\section{BIBLIOGRAPHIE}

ALEKSEENKo Evgenija Alekseevna, 1984, «Južnosibirskie paralleli v šamanstve ketov », in

Etnografija Narodov Sibiri, Novossibirsk, Nauka, p. 77-83.

Avvakum, 1938, La vie de l'archiprêtre Avvakum écrite par lui-même. Trad. Pierre Pascal, Paris, Gallimard.

DELABY Laurence, 1998, « Pas de chapeau à queues pour la chamanesse : aperçu sur le rôle du clan dans le chamanisme golde ", Études mongoles... et sibériennes, 29, p. 61-79.

FRACHETTI Michael D., 2012. « Multiregional Emergence of Mobile Pastoralism and Non Uniform Institutional Complexity across Eurasia », Current Anthropology, 53 (1), p. 2-38.

FRANCFORT Henri-Paul, 2001, «Art, Archaeology and the Prehistories of Shamanism in Inner Asia ", in Francfort H.-P., Hamayon R. N. (éds.), The Concept of Shamanism: Uses and Abuses, Budapest, Akadémiai Kiadó, p. 243-276.

GoLOVNEV Andrej, 2004, Kočeniki tundry: nency i ih fol'klor, Ekaterinburg, Etnografičeskoe bjuro.

HAMAYON Roberte, 1990, La Chasse à l'âme. Esquisse d'une théorie du chamanisme sibérien, Nanterre, Société d'Ethnologie.

-, 2001, « Shamanism: Symbolic System, Human Capability and Western Ideology », in Francfort H.-P., Hamayon R. N. Hamayon (éds)., The Concept of Shamanism : Uses and Abuses, Budapest, Akadémiai Kiadó, p. 1-27.

-, 2007, « À quel esprit se vouer ? Vicissitudes de la notion d'esprit chamanique en Sibérie postsoviétique », La nature des esprits dans les cosmologies autochtones. Nature of spirits in aboriginal cosmologies, p. 403-425.

HEISSIG Walther, 1987, « Kalevalan ja Mongolieeposteen yhteset motiivit », Kalevala Ja Maailman Eepokset, 16, p. 337-346.

Humphrey Caroline, 1999, «Shamans in the City », Anthropology Today, 15 (3), p. 3-10.

IDES Evert Ysbrants, 1704, Three Years Travels from Moscow over-Land to China: Thro' Great Ustiga, Siriania, Permia, Sibiria, Daour, Great Tartary, Etc. to Peking, Londres,Freeman.

JACOBSON Esther, 2001, « Shamans, Shamanism and Anthropomorphizing Imagery in Prehistoric Rock Art of the Mongolian Altai », in Francfort H.-P., Hamayon R. N. (éds.), The Concept of Shamanism: Uses and Abuses, Budapest, Akadémiai Kiadó, p. 277-296.

-, 2004. « Ancient North Asian Shamanism », in Walter M., Neumann Fridman E. (éds.), Shamanism. An Encyclopedia of World Beliefs, Practices, and Culture, Santa Barbara, Abc-Clio, p. 532-536.

Jochelson Waldemar, 1926, The Yukaghir and the Yukaghirized Tungus, Leiden, New York, E. J. Brill Ltd. ; G. E. Stechert.

JoHnson Richard, 1886, « Certaine Notes Unperfectly Written by Richard Iohnson Seruant to Master Richard Chancelour, Which Was in the Discouerie of Vaigatz and Noua Zembla, with Steuen Burrowe in the Serchthrift 1556 and Afterwarde among the Samoedes, Whose Deuilish Rites Hee Describeth », in Hakluyt R. (éd.), The Principal Navigations, Voyages, Traffiques and Discoveries of the English Nation, Goldsmid, $3: 138-41$, Edinbourg. 
KYZLASOv Leonid Romanovič, 1952, « Drevnejšee svidetel'stvo ob olenevodstve », Sovetskaja Ètnografija 2, p. 39-49.

Lopatin I. A., 1960, The Cult of the Dead among the Natives of the Amur Basin, La Haie, 'S-Gravenhage, Mouton.

LUNEAU Élise, 2013, « Nomades et sédentaires en Asie Centrale. À l'âge du Bronze », in Stépanoff C., Ferret C., Lacaze G., Thorez J. (éds.), Nomadismes d'Asie Centrale et Septentrionale, Armand Colin, Paris, p. 232-34.

MachiceK M. L., Zubova A. V., 2012, « Dental Wear Patterns and Subsistence Activities in Early Nomadic Pastoralist Communities of the Central Asian Steppes », Archaeology, Ethnology and Anthropology of Eurasia, 40, p. 149-157.

MERLi Laetitia, 2010, De l'ombre à la lumière, de l'individu à la nation : ethnographie du renouveau chamanique en Mongolie postcommuniste, Paris, EPHE.

NомокоNOVA Tatiana et al., 2010, « Late Holocene subsistence practices among Cis-Baikal pastoralists, Siberia: zooarchaeological insights from Sagan-Zaba II », Asian perspectives, 49 (1), p. 157-179.

Pedersen Morten Axel, 2011, Not Quite Shamans: Spirit Worlds and Political Lives in Northern Mongolia. Culture and Society after Socialism, Ithaca, Cornell University Press.

PotAPov Leonid Pavlovič, 1953, « Pišča Altaicev », Sbornik Muzeja Antropologii i Ètnografii, 14, p. 37-71.

-, 1991, Altajskij šamanizm. Leningrad, Nauka.

RøED, KNUT H. et al., 2008, « Genetic Analyses Reveal Independent Domestication Origins of Eurasian Reindeer », Proceedings of the Royal Society B : Biological Sciences, 275 (1645), p. 1849-1855.

SHIROKOGOROFF S. M., 1935, Psychomental Complex of the Tungus, London, Kegan Paul, Trench, Trubner \& Co.

STÉPANOFF, Charles, 2012, « Human-Animal “joint Commitment” in a Reindeer Herding System, Hau, Journal of Ethnographic Theory, 2 (2), p. 287-312.

-, 2013, « Dessins chamaniques et espace virtuel chez les Khakasses », Gradhiva, Revue d'anthropologie et d'histoire des arts, 17, p. 144-69.

-, 2014, Chamanisme, rituel et cognition chez les Touvas (Sibérie du Sud), Paris, Maison des sciences de l'homme.

StÉPANOFF Charles, FERRET Carole, LACAZE Gaëlle, THorEZ Julien (éds.), 2013, Nomadismes d'Asie centrale et septentrionale, Paris, A. Colin.

SvitKova V. S. (éd.), 1993, Istorija Hakasii. S drevnejših vremen do 1917, Moscou, Nauka.

TAKAKURA Hiroki, 2012, « The Shift from Herding to Hunting among the Siberian Evenki: Indigenous Knowledge and Subsistence Change in Northwestern Yakutia (English) », Asian Ethnology, 71 (1), p. 31-47.

VASILEVIČ Glafira Makar'evna, 1969, Evenki. Istoriko-etnografičeskie očerki (XVIII-načalo XX v.), Leningrad, Nauka.

VAsiLEvič Glafira Makar'evna, Levin M. G., 1951, « Tipy olenevodstva i ih proishoždenie », Sovetskaja Etnografija, 1, p. 63-87. 


\section{NOTES}

1. http://assr.revues.org/25629

2. Je remercie Isabelle Charleux, Grégory Delaplace et Thierry Zarcone d'avoir lu et commenté ce texte, dont la teneur n'engage que moi.

\section{AUTEUR}

\section{CHARLES STÉPANOFF}

École pratique des hautes études, Laboratoire d'anthropologie sociale 